\title{
Evaluation of the PGP3 ELISA for surveillance of the burden of Chlamydia in Australia and Samoa
}

Rami Mazraani ${ }^{1}$, Peter Timms ${ }^{2}$, Phillip Hill ${ }^{3}$, Sailau Sauulii-Saunii ${ }^{4}$, Liai Iosefa-Siitia ${ }^{5}$, Tavita Niupulusu ${ }^{6}$, Seiuli V. A. Temese ${ }^{7}$, Liai Iosefa-Siitaia ${ }^{8}$, Leveti Auvaa ${ }^{9}$, Siuomatautu A. Tapelu ${ }^{6}$, Maauga Motu ${ }^{8}$, Antoinette Righarts ${ }^{10}$, Michael S. Walsh ${ }^{11}$, Luk Rombauts ${ }^{12}$, John A. Allan ${ }^{13}$, Patrick Horner ${ }^{14}$, Wilhelmina M. Huston ${ }^{1}$

\begin{abstract}
Serological assays could be used to investigate the population burden of infection and potentially sequelae from Chlamydial infection or subfertility in Australian and Samoan women. The PGP3 ELISA was conducted on 559 serum samples and the absorbance's compared to women with infertility, sub-fertility and fertile women. In Australia chlamydial tubal factor infertility had significantly higher absorbance's in the PGP3 ELISA compared to women with other forms of infertility $(\mathrm{p}<0.008)$, fertile $(\mathrm{p}<0.007)$, but not current infection $(\mathrm{p}=1.0)$ (all adjusted for age). In Samoa, there were no significant differences in the PGP3 ELISA absorbance between chlamydial subfertile women and women with a current infection and fertile women. The two populations have significantly different overall absorbance's in the ELISA $(\mathrm{P}<0.0001)$.
\end{abstract}

Introduction: Chlamydia trachomatis is the most common bacterial sexually transmitted infection worldwide. Ades et al., 2017 evaluated whole organism immunofluorescence titres (WIF) from 1985-1995 for women undergoing care for tubal factor infertility. And estimated the fraction of chlamydial related tubal factor infertility (TFI) to be a minimum of $28 \%$. Numerous studies have shown that chlamydial sero-positivity (typically measured by higher titres), in infertile women has been significantly associated with laparoscopically-diagnosed TFI. Recent studies in the UK have demonstrated that the Pgp3 ELISA could be used for such monitoring and evaluating.

Method: We tested for a significantly higher absorbance in groups
categorised by fertility and infection status in a PGP3 ELISA. Groups of
women as previously described were categorised using surgery or
epidemiological tools as well as NAAT results. Two separate groups
recruited at fertility treatment clinics (Group $1: \mathrm{n}=97$ and Group $4: \mathrm{n}=73$ )
categorised for the purpose of this study by tubal status and serological
results, a fertile control group (Group $2: \mathrm{n}=53$ ), and acute NAAT confirmed
chlamydial infection group (Group 3: $\mathrm{n}=79$ ).
Group $5(\mathrm{n}=239$ ) were from a previously described cross-sectional sero-
epidemiological study in Samoa.

Table 1. PGP3 ELISA absorbance in subfertile, infected and fertile groups in Australia and Samoa

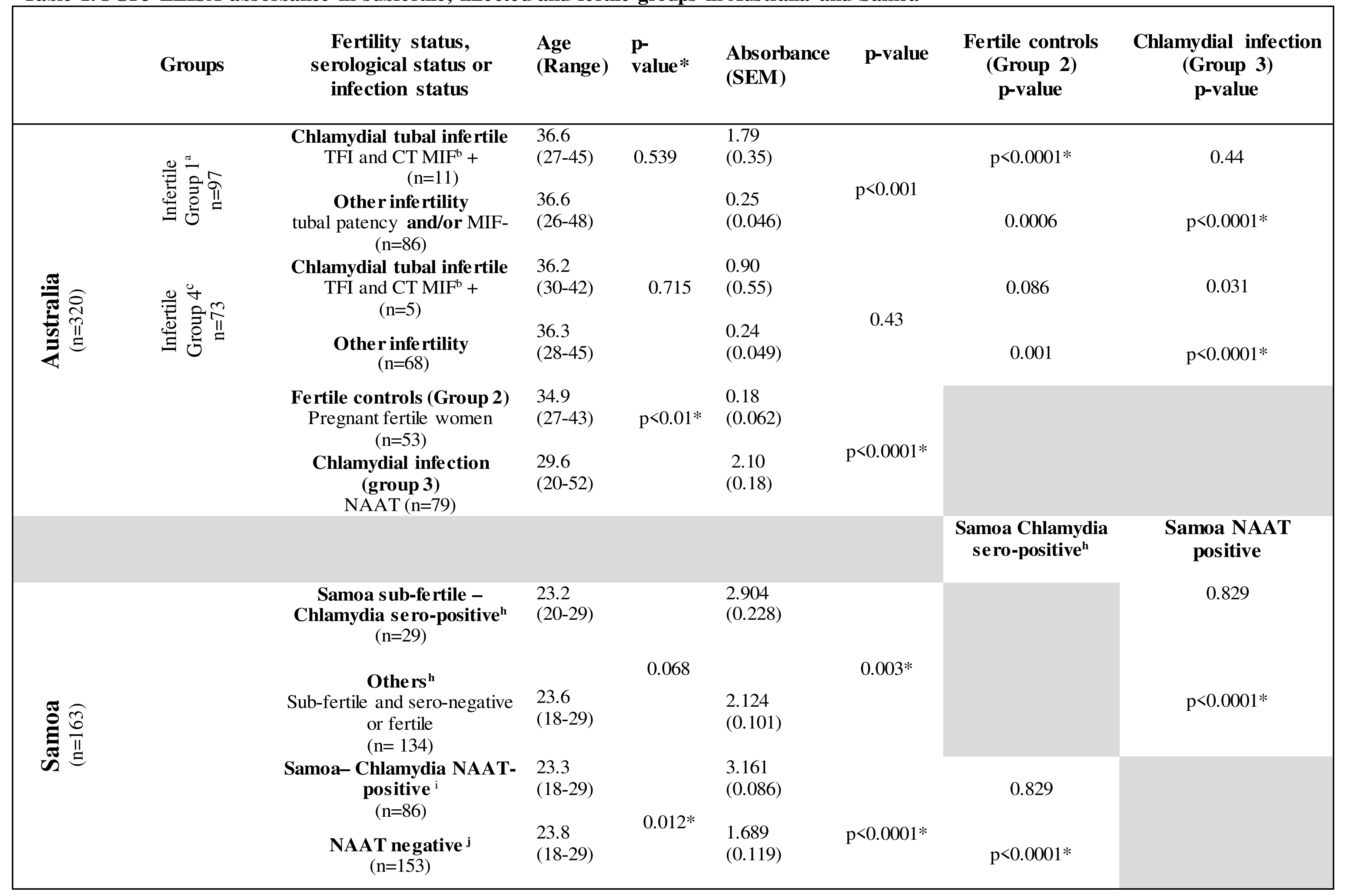

Conclusion:The data presented here means we now have a global dataset (in conjunction with the recent UK studies supporting that the Pgp3 ELISA has potential for sero-epidemiological studies. We propose longitudinal sero-epidemiological monitoring of public health intervention programs . 